\title{
Visualisation of the Vocal-Tract Shape for a Computer-Based Speech Training System for the Hearing-Impaired
}

\author{
A.E. Mahdi* \\ Department of Electronic and Computer Engineering, University of Limerick, Limerick, Ireland
}

\begin{abstract}
A computer-based tool for visualisation of the vocal-tract, during speech articulation, by means of a midsagittal view of the human head has been designed and developed. The vocal tract graphics are generated using a new approach that is based on estimating both the area functions and the formant frequencies from the acoustic speech signal. First, it is assumed that the speech production process is an autoregressive model. Using a linear prediction analysis, the vocal tract area functions and the first three formants are estimated. The estimated area functions are then mapped to corresponding mid-sagittal distances and displayed as 2D vocal tract lateral graphics. The mapping process is based on a simple numerical algorithm and an accurate reference grid derived from $\mathrm{x}$-rays for the pronunciation of a number English vowels uttered by different speakers. To compensate for possible errors in the estimated area functions due to variation in vocal tract length between speakers, the first two sectional distances are determined by the three formants. Experimental results show high correlation with x-ray data and the PARAFAC analysis. The tool also displays other speech parameters that are closely related to the production of intelligible speech and hence would be useful as a visual feedback aid for speech training of hearing-impaired individuals and children with speech impediment.
\end{abstract}

\section{INTRODUCTION}

The process of learning to speak in the case of people with normal hearing is primarily aided by auditory feedback. However, for those who suffer from deafness, learning to speak naturally is a very difficult process. With limited auditory capability, a hearing-impaired person often lacks models of speech targets necessary to produce normal speech. In an effort to overcome this difficulty, many attempts have been made to provide a substitute for the feedback mechanism with visual speech display devices [1-6]. However, without any articulatory correlate, the benefits of such devices were limited. In order to produce a natural and intelligible speech, a speaker needs to know how to use the vocal organs in regards to correct position of the articulators, breathing, loudness, rhythm and nasalization [6,7]. Hence the availability of visual information regarding these aspects would greatly help the hearing-impaired improving their speaking abilities.

This paper describes a system which visualises a speaker's vocal tract by means of mid-sagittal graphical plots of the human head. The vocal tract shapes, and other related speech parameters, are graphically displayed on a PCmonitor using information extracted directly from the acoustic speech signal as picked up by a microphone or loaded from an audio file. To estimate the necessary parameters, it is assumed that the speech production process is an autoregressive (AR) model. The vocal tract area functions, log spectra and the first three formants are then estimated, by employing a linear prediction (LP) analysis, and used to construct the corresponding vocal tract graphics and other parameters display.

*Address correspondence to this author at the Department of Electronic \& Computer Engineering, University of Limerick, Limerick, Ireland; Tel: (+)353-61-213492; Fax: (+)353-61-338176; E-mail: Hussain.Mahdi@ul.ie

\section{SPEECH ANALYSIS MODEL}

Speech is the acoustic wave that is radiated from the vocal system when air is expelled from the lungs and the resulting flow of air is perturbed by a constriction somewhere in the vocal tract. This speech production process can be practically modelled using the well-known all-pole source-filter approach, which represents the speech signal in terms of an AR model [8]. According to this model, speech is split into a rapidly varying excitation signal, generated by an impulse train input or a random noise generator, and a slowly varying filter representing the vocal tract. Voiced speech is produced by taking the impulse train as excitation. In unvoiced segments, the random white noise is used as the excitation. The output speech is produced by passing the excitation through the vocal tract filter. Hence, changes in the vocal tract configuration, reflected by the filter, produces corresponding changes in the spectral envelope of the speech signal. Therefore to estimate the vocal tract shape form the speech signal, an inverse filter model has to be used $[9,10,11]$.

The speech analysis model used in this work is shown schematically in Fig. (1). In this model, it is assumed that the speech to be analysed is limited to periodic non-nasalised voiced sounds so that the filter in Fig. (1) is driven by an impulse train. This means that the filter includes all the contributions from the glottal wave, the vocal tract and the radiation impedance at the lips. The inverse filter is assumed to be a linear filter with only zeros in its transfer function, and the power spectral envelope of the speech is assumed to be approximated by poles only. Accordingly, the transfer function of the inverse filter can be expressed in terms of $\mathrm{z}$ transform notation as:

$$
A(z)=\sum_{i=0}^{M} a_{i} z^{-i}, \quad a_{0}=1
$$


where $a_{i}$ are the coefficients of the inverse filter, $M$ is the order of the filter, $z=e^{j \omega T}$ and $T$ is the sampling period. Here, $a_{0}$ affects only the gain of the system, hence no generality is lost by setting $a_{0}=1$. The objective of this analysis is to obtain a close representation of the vocal tract. To obtain this representation, one needs to estimate the coefficients of the optimal inverse filter described by equation (1). However, it can be shown that $A(z)$ is also an inverse transfer function of a non-uniform acoustic tube model of the all-pole vocal tract model [12]. Thus the optimal inverse filter process in the above speech analysis model can be equivalently replaced by a filtering process of an acoustic tube of length $l$, which is the assumed length of the vocal tract, divided into arbitrary number, $M$, of sections with equal length $\Delta l$, provided that [12]:

a) The continuity conditions for the volume velocity and sound pressure are satisfied at each junction between two adjacent sections;

b) The length of the individual tube sections, $\Delta l$, are kept short compared to the wavelength at the highest frequency of interest;

c) No losses are accounted for, and

d) It should also be noted that the identity of the filtering process of the above described acoustic tube to that of the optimum inverse filter is obtained under the condition $z=e^{j \omega 2 \Delta l / c}=e^{j \omega T}$, where $c$ is the velocity of sound. Consequently, the sampling frequency $F_{s}=1 / T$ and the number of sections $M=l / \Delta l$ is constrained by [12]:

$F_{s}=\frac{M c}{2 l}$

Therefore, as long as $F_{s}$ is constant, the vocal tract length, $l$, is assumed to be fixed here and $M$, i.e. number of the sections or the filter's order, has to be chosen to satisfy equation (2).

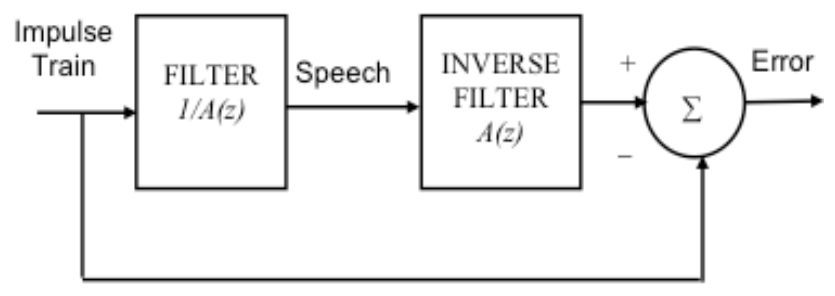

Fig. (1). Speech analysis model used in the system.

\section{MODELLING THE VOCAL TRACT}

The vocal tract can be modelled as an acoustic tube of varying diameter. If we abstract from the vocal tract curvature, the acoustic tube can be divided into cylindrical sections of equal lengths. Depending on the shape of the acoustic tube, a sound wave travelling through it will be reflected in a certain way so that interferences will generate resonances at certain frequencies. These resonances are called formants. Their location largely determines the speech sound that is heard.
It is well known that the linear prediction (LP) analysis of speech signals is based on an AR speech production model [10]. It has also been shown by several researchers that the LP process is equivalent to the filtering process of a non-uniform acoustic tube model where the tube is divided into an arbitrary number of sections of equal length [12-14]. Thus, if the conditions stated at the end of Section 2 are satisfied, and if the speech signal is pre-emphasis to compensate for the spectral characteristics of the glottal excitation source and for the lips radiation impedance, then estimates of the vocal tract area functions can be obtained by computing the reflection coefficients at the junctions between adjacent sections of the equivalent acoustic tube. This can be done by using an LP model of the appropriate order and the following relation:

$\mu_{i}=\frac{A_{i+1}-A_{i}}{A_{i+1}+A_{i}} \Leftrightarrow A_{i}=A_{i+1} \frac{1-\mu_{i}}{1+\mu_{i}}$

where $A_{i}$ and $A_{i+1}$ are the cross-sectional areas of two adjacent sections of the non-uniform acoustic tube indexed in ascending order from the lips to the glottis, and $\mu_{i}$ is the reflection at the junction between these two sections.

\section{SYSTEM DESCRIPTION AND DESIGN}

Using the vocal tract model described in Section 3, a PCbased prototype system for visualisation of the human vocal tract shapes and other associated speech parameters has been designed and developed. The system uses the PC's sound card operating with $8 \mathrm{kHz}$ sampling frequency and 16-bit resolution, to extract the necessary speech parameters directly from the acoustic speech waveform. The block diagram given in Fig. (2) depicts the functionality and main processing blocks of the developed system.

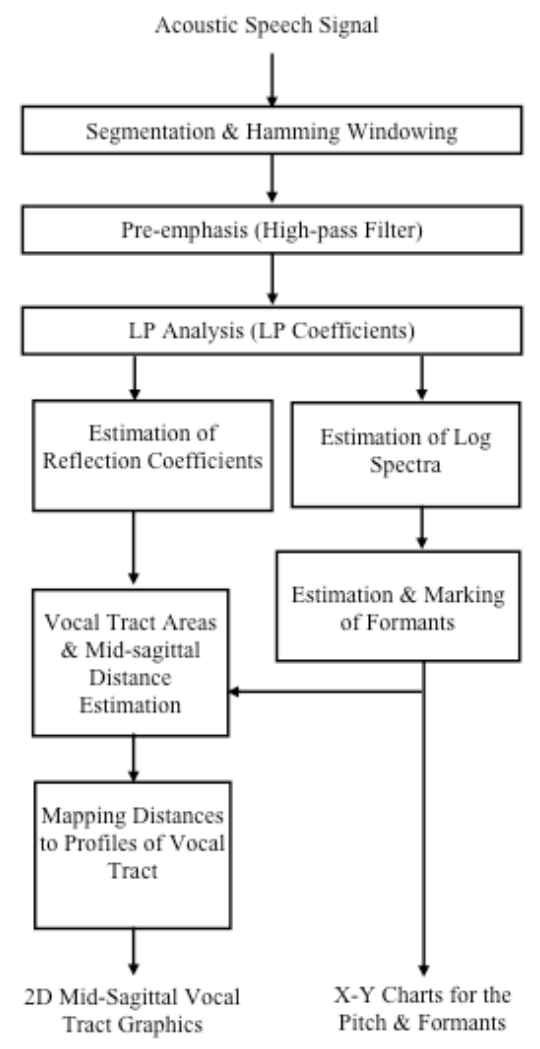

Fig. (2). Functional block diagram of developed system. 
For simplicity, our system uses an initial assumption that the vocal tract is $17 \mathrm{~cm}$ long. As the PC's sound card samples the speech at the rate of $8 \mathrm{kHz}$, thus satisfying equation (2) requires $M$ to be equal to 8, i.e. the initial use of an acoustic tube with 8 sections. Consequently, an $8^{\text {th }}$ order LP analysis model is employed by the system.

\section{Estimation of the Area Functions}

The speech signal is segmented into $30 \mathrm{~ms}$ frames using a hamming window of an appropriate length. A preemphasis of an approximately $6 \mathrm{~dB} /$ octave is applied to the current frame using an FIR high-pass filter of the form:

$$
y_{n}=x_{n}-0.9375 x_{n-1}
$$

The reflection coefficients are computed by applying an $8^{\text {th }}$ order LP analysis model using an autocorrelation method that uses LPC uses the Levinson-Durbin recursive algorithm due to its simplicity and ease of its implementation within the chosen computing environment. Equation (3) is then used to estimate the corresponding vocal tract area functions as discussed in Section 3. As an example, Fig. (3) (the upper half) shows the normalised area functions for the English vowel $/ \mathrm{UH} /$, as computed by the system using an 8 -section acoustic tube model. The LP model is also used to obtain the log spectra, whose peaks are then marked to identify and estimate the first three formants, as also illustrated in lower half of Fig. (3).

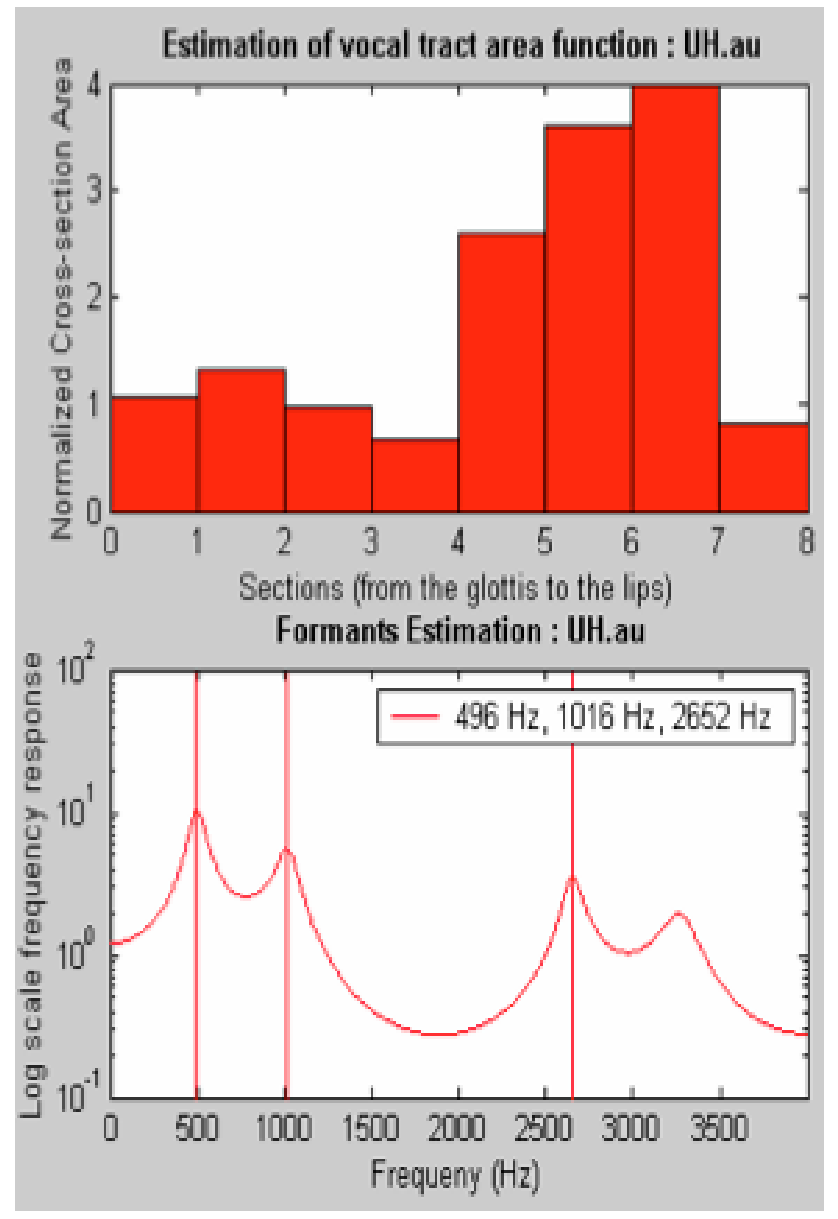

Fig. (3). Vocal tract area functions and the first three corresponding formants for the vowel /UH/.

\section{Mapping to Mid-Sagittal Distances}

Since the human vocal tract does not resemble an exact circular tube, there is therefore a need to modify the above computed area functions such that they map correctly into mid-sagittal distances of the vocal tract profiles. Several areas to profile transformation techniques have been developed $[12,14]$. Most such techniques rely on derivation of suitable application-specific transformation parameters using complex analysis of $\mathrm{x}$-ray and cine-fluorograms of various speakers. A common technique is the $\alpha \beta$ model [12], which is described by:

$$
A_{i}=\alpha_{i} d_{i}^{\beta_{i}} \Leftrightarrow d_{i}=\left(\frac{A_{i}}{\alpha_{i}}\right)^{1 / \beta_{i}}
$$

where $A_{i}$ is the cross-sectional area of a given section, $d_{i}$ is the mid-sagittal distance and $\alpha_{i}$ and $\beta_{i}$ are section dependent parameters. In our system, we employ a new method based on the above model to measure the mid-sagittal distances along the lines of a semipolar grid (See Fig. 4) according to the following procedure:

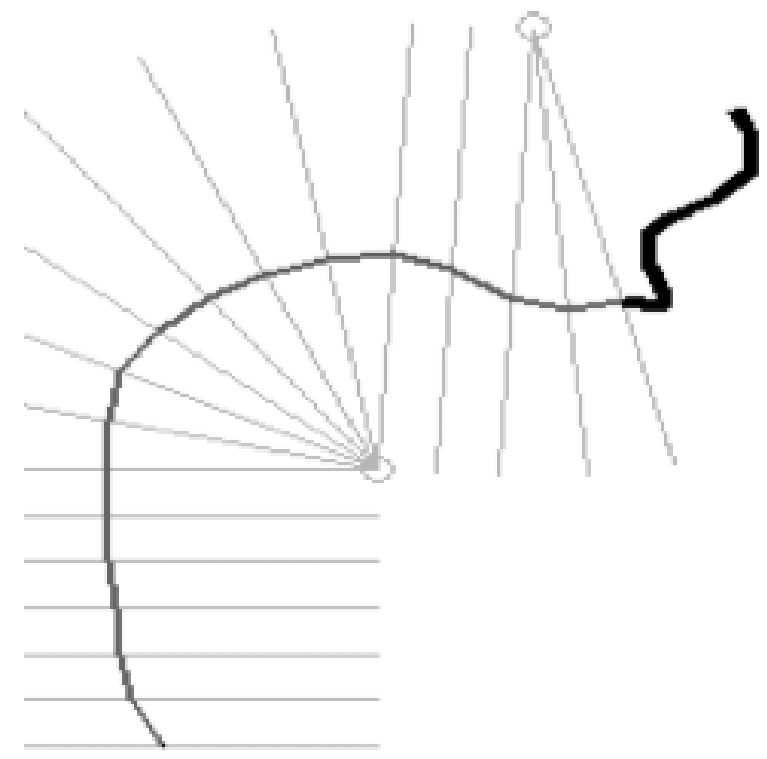

Fig. (4). The reference grid of the upper jaw as used in the system.

a) The vocal tract was assumed to be divided into 18 equal sections.

b) In the vocal organs, the shortest path from the upper to the lower part of each section was selected.

c) The upper jaw was assumed to be fixed and the lower jaw was movable.

d) A reference grid for the upper jaw based on x-ray data of the lateral shape of the vocal tract and on results of the PARAFAC analysis [16] was designed, as shown in Fig. (4). In this grid, straight perpendicular lines were drawn through the centre of each section, in accordance with (b) above.

e) The 8 area functions estimated by the 7 th order LP model were re-sampled and redistributed to fit the 18section vocal tract configuration used in the system. 
f) Based on equation (5), a simple numerical procedure is used to estimate the values of the coefficients $\alpha$ and $\beta$ that minimize the root mean squared error between the area functions estimated in (e) above and those derived from measurement data obtained from [15] for pseudosagittal dimensions of the tongue position for five speakers each saying ten English vowels. The estimated area functions are then interrupted as functions of $\alpha$ and $\beta$, as given in equation (5), to compute the midsagittal distances.

As indicated previously, initially the vocal tract is modelled in our system with an assumption that it is $17 \mathrm{~cm}$ long. It is known, however, that the vocal tract length for the utterance of various sounds varies even for a single speaker. In addition, the male vocal tract is generally slightly longer than $17 \mathrm{~cm}$, while children and females have shorter vocal tracts [16]. Hence, the above assumption may cause an error in the distribution of the area functions along the assumed vocal tract configuration. To compensate for this possible source of error, the first two mid-sagittal distances have been determined from the three estimated formants $F_{1}, F_{2}$ and $F_{3}$ as follows [17]:

$$
X_{1}=C_{1} F_{2}+C_{2} F_{2} F_{3}+C 3 F_{1} / F_{2}+C_{4}
$$

where $X_{1}$ is the mid-sagittal distance between the lips in $\mathrm{cm}$, $C_{1}=0.3 \times 10^{-3}, C_{2}=-0.343 \times 10^{-6}, C_{3}=4.143, C_{4}=-2.865$. The mid-sagittal distance between the upper and lower teeth, $X_{2}$, is estimated by:

$$
X_{2}=\frac{X_{1}+X_{3}}{2}
$$

where $X_{3}$ is the mid-sagittal distance extracted from the vocal tract area function that corresponds to section 3 . In addition, the estimated formants have been used to adjust the rounding degree of the lips and the height of the jawbone on the designed vocal tract lateral graphics.

\section{RESULTS AND DISCUSSION}

The vocal tract visualisation tool has been designed to operate with MS Windows-based PC environment. The multi-display window and other user's features of the complete system are shown in Fig. (5). As can be seen, the system's screen is divided into four windows for displaying the vocal tract graphics, the sound intensity, the pitch and the first three formants of the speech signal. The system can operate in two main modes: (a) near real-time mode, whereby the speech signal is picked up by a microphone connected to the PC sound card (as with the case shown in

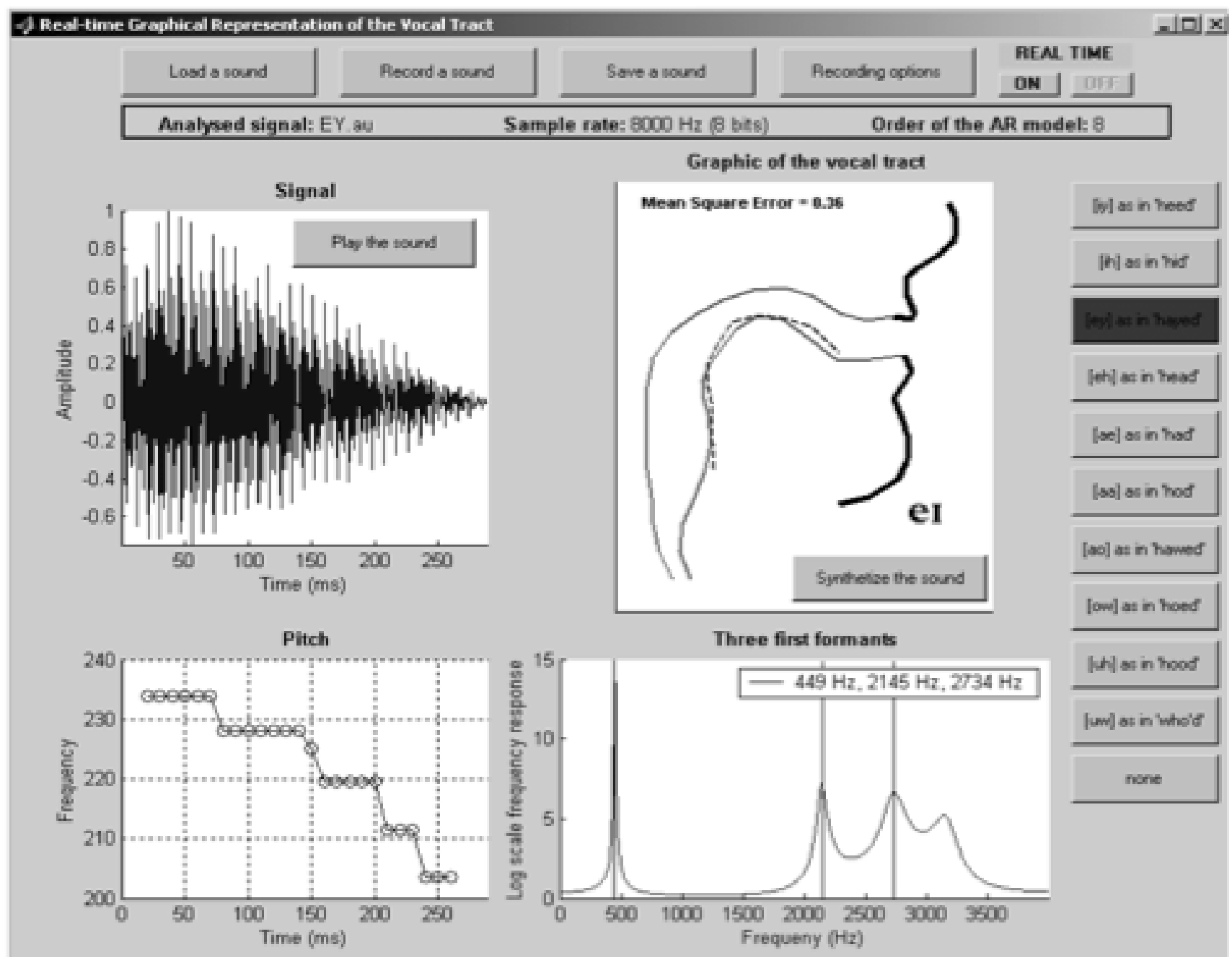

Fig. (5). System's multi-pane screen display and user's extracted features. 
Fig. 5), and (b) non real-time mode, whereby the speech signal is either recorded by the system or read from a stored audio file, and its features are then displayed. It also allows the saving of speech/sound signals. For the vowel articulation, the user can compare the shape of his/hers vocal tract to a reference trace (shown with a dashed line in Fig. 5) for the correct tongue position derived from the measurements data reported in [9]. The deviation from the reference trace is given for this case in the form of a computed mean squared error (MSE) of all the estimated mid-sagittal distances.

Fig. (6) shows the vocal tract profiles for 10 American English vowels, as estimated by the system (dashed lines represent reference trace for tongue position). For comparison and evaluation purposes, the deviations, in terms of MSE values, from the reference tongue position data adopted from [15] are also indicated. In general, the obtained results seem to correlate well with the reference data. They were also found to correlate well with $x$-ray data and the PARAFAC analysis. Referring to the MSE values shown in Fig. (6), the system seems to perform particularly well in the cases of all the 'front vowels', such as /IY/, /EY/, /IH/, /EH/ and /AE/, with the MSE increasing as the vowel height decreases. With the exception of /AA/ and / $\mathrm{UH} /$, the results show relatively less accurate correlation with the reference data for the cases of the 'back vowels'. As vowel classification into front and back vowels is related to the position of the tongue elevation towards the front or the back of the mouth, we believe that the higher accuracy in the cases of the front vowels is attrib- uted to the formant-based added adjustments of the lips, jawbone and front sections of the vocal tract we used in our approach.

On the other hand, the relative length of the vowel's vocalisation seems to affect the accuracy of the estimated area functions and hence the displayed vocal tract shape. In specific, the system seems to give relatively lower accuracy for relatively longer vowels, such as /AO/, and complex vowels which involve changes in the configuration of the mouth during production of the sound, such as /OW/. We believe this is due to the fact that the system, in its current design, bases its estimation of the speech parameters on information extracted from the 2-3 middle frames of the analysed speech waveform.

\section{CONCLUSIONS}

We have described the process of designing and development of a computer-based system for the near real-time and non real-time visualisation of the vocal tract shape during vowel articulation. Compared to other similar systems, our system uses a new approach for estimating the vocal tract mid-sagittal distances based on both the area functions and the first three formants as extracted from the acoustic speech signal. It also utilises a novel and simple technique for mapping the extracted information to corresponding midsagittal distances on the displayed graphics. The system is also capable of displaying the sound intensity, the pitch and the first three formants of the uttered speech. It extracts the

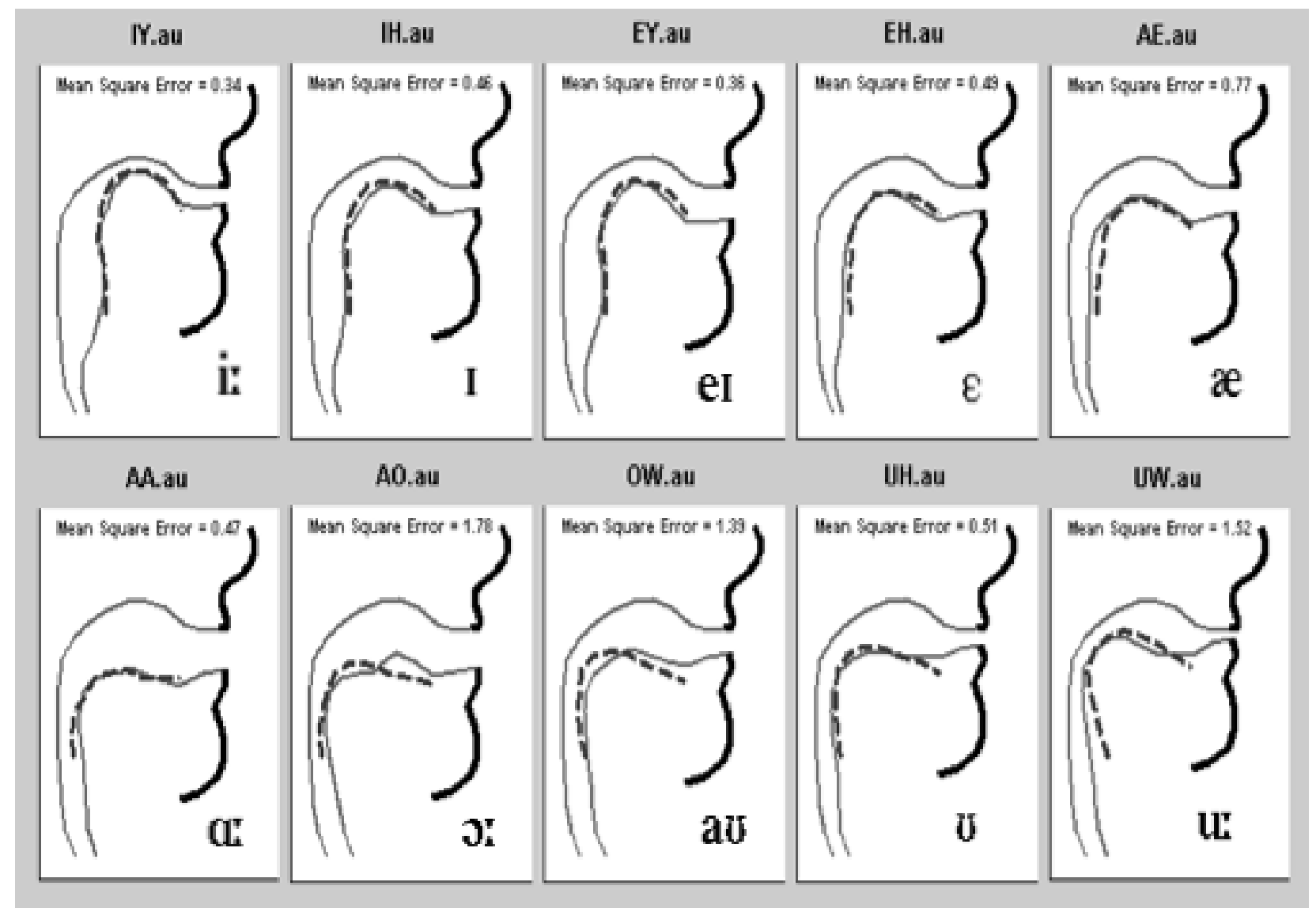

Fig. (6). Vocal tract profiles for 10 American English vowels as estimated by the system (dashed lines represent reference traces for tongue positions). 
required parameters directly from the acoustic speech signal using an AR speech production model and LP analysis. Reported preliminary experimental results have shown that in general the system is able to reproduce well the shapes of the vocal tract, with real-time sensation, for vowel articulation. Work is well underway to optimise the algorithm used for extraction of the required acoustics information and the mapping technique, such that dynamic descriptions of the vocal tract configuration for long and complex vowels, as well as vowel-consonant and consonant-vowel are obtained. Enhancement of the system's real-time capability and features, and facilitation of an integrated speech training aid for the hearing-impaired are also being investigated.

\section{REFERENCES}

[1] Y. Yamada , H. Javkin, and K. Youdelman, "Assistive speech technology for persons with speech impairments“, Speech Comm., vol. 30, issue 2; pp. 179-187, 2000

[2] C. D. Choi. "A Review on Development of Visual speech Display Devices for Hearing Impaired Children“, Commun. Disorders, vol. 5, issue 2; pp. 38-44, 1982.

[3] H. T. Bunnell, D. Yarrington, and D. M. Polokoff, "STAR: articulation training for young children", in Intl Conf on Spoken Language Processing (ICSLP/INTERSPEECH), 2000, vol. 4, pp. 85-88.

[4] J. J. Mashie. "Use of sensory aids for teaching speech to children who are deaf", in Profound Deafness and Speech Communication, K-E. Spens and G. Plant, Eds. London: Whurr Publishers Ltd, 1995, pp. 61-491.

[5] M-L. Hsiao, P. T. Li, P. Y. Lin, S-T. Tang, T-C. Lee, and S-T. Young, "A computer based software for hearing impaired children's speech training and learning between teacher and parents in Taiwan", in 23rd Annual Int. Conf. of the IEEE Engineering in Medicine and Biology Society, 2001, vol. 2, pp.1457- 1459.

[6] A-M. Öster, "Clinical applications of computer-based speech training for children with hearing-impairment", in 4th International
Conference on Spoken Language Processing (ICSLP), 1996, pp. 157-160.

[7] E. Eriksson, O. Balter, O. Engwall, A-M. Oster, and H. Sidenbladh-Kjellstrom, "Design recommendations for a computerbased speech training system based on end-user interviews", in Intl Conf on Speech and Computer (SPECOM), 2005, pp. 483-486.

[8] P. S. R. Diniz, E. A. B. da Silva, S. L. Netto, Digital Signal Processing: System Analysis and Design. Cambirdge: Cambridge University Press, 2002.

[9] A. Toutios, "Acoustic-to-articulatory inversion of speech and detection of phonetic features in the speech signal", Student Talk at the Machine Learning Summer School (MLSS), Tuebingen, Germany, 2003. (avaialble at: http://macedonia.uom.gr/ toutios/mlss.htm)

[10] T. E. Quatieri, Discrete-time Speech Signal Processing, Principles and Practice. NJ: Prentice Hall, 2002.

[11] H. Yehia, and F. Itakura, "A method to combine acoustic and morphological constraints in the speech production inverse problem“, Speech Comm., vol. 18, issue 2. pp.151-174, 1996.

[12] S. Krstulovic, "Relating LPC modeling to a factor-based articulatory model", in Intl Conf on Spoken Language Processing (ICSLP), 2000, vol. 3, pp. 67-70.

[13] H. Deng, M. P. Beddoes, R. K. Ward, and M. Hodgson, "Estimating the vocal-tract area function from a speech signal", in Proc. of Canadian Acoustic Week, Edmonton, Canada, 2003, pp.40-41.

[14] A. Soquet, V. Lecuit, T. Metens, and D. Demolin, "Mid-sagittal cut to area function transformations: direct measurements of midsagittal distance and area with MRI“, Speech Comm., vol. 36, issue 3, pp.169-180, 2002.

[15] R. Harshman, P. Ladefoged, and L. Goldstein, "Factor analysis of tongue shapes", J. Acoustic. Soc. Am., vol. 62, issue 3, pp. 693-706, 1977.

[16] B. F. Necioğlu, M. A. Clements, T. P. Barnwell III, "Unsupervised estimation of the human vocal tract length over sentence level utterances", in IEEE Intl Conf on Acoustics, Speech Sig Procss (ICASSP), 2000, vol. 3, pp.1319-1322.

[17] T. Gay, L-J. Boé L-J, and P. Perrier, "Acoustic and perceptual effects of changes in vocal tract constrictions for vowels", $J$. Acoustic. Soc. Am., vol. 92, issue 3, pp.1301-1309, 1992.

(C) A.E. Mahdi; Licensee Bentham Open.

This is an open access article distributed under the terms of the Creative Commons Attribution License (http://creativecommons.org/license/by/2.5/), which permits unrestrictive use, distribution, and reproduction in any medium, provided the original work is properly cited. 